\title{
Bounds on the smallest eigenvalue of finite element equations with adaptive (anisotropic) meshes without regularity assumptions
}

\author{
Lennard Kamenski* \\ *m4sim GmbH, Berlin, Germany \\ e-mail: lkamenski@m4sim.de, web page: https://m4sim.de
}

\begin{abstract}
Highly adapted or misshaped meshes can have a strong influence on the conditioning of the finite element (FE) equations, which, in turn, has an impact on convergence properties and accuracy of iterative methods for solving the resulting linear systems. In most of the available work, some local or global mesh regularity properties are required in order to bound the constants during the derivation of the estimates, which causes degeneration of these estimates in case of extreme mesh geometries or strong irregular anisotropic adaptation.
\end{abstract}

The provided bound [2] for the smallest eigenvalue of the FE equations has a similar form as the one by Graham and McLean [1] but doesn't require any mesh regularity assumptions, neither global nor local. In particular, it is valid for highly adaptive, anisotropic, or non-regular meshes without any restrictions.

In three and more dimensions, the bound depends only on the number of degrees of freedom $N$ and the Hölder mean $M_{1-d / 2}\left(|\tilde{\omega}| /\left|\omega_{i}\right|\right)$ taken to the power $1-2 / d$,

$$
\lambda_{\min }(A) \gtrsim N^{-1} \times M_{1-d / 2}^{1-2 / d}\left(\frac{|\tilde{\omega}|}{\left|\omega_{i}\right|}\right),
$$

where $M$ denotes the Hölder mean $M_{p}\left(x_{1}, \ldots, x_{n}\right)=\left(\frac{1}{n} \sum_{i=1}^{n} x_{i}^{p}\right)^{1 / p}$, and $|\tilde{\omega}|$ and $\left|\omega_{i}\right|$ the average mesh patch volume and the volume of the patch corresponding to the $i^{\text {th }}$ mesh node, respectively.

In two dimensions, the bound depends on the number of degrees of freedom $N$ and the logarithmic term $\left(1+\left|\ln \left(N\left|\omega_{\min }\right|\right)\right|\right)$, where $\left|\omega_{\min }\right|$ denotes the volume of the smallest patch,

$$
\lambda_{\min }(A) \gtrsim N^{-1} \times\left(1+\left|\ln \left(N\left|\omega_{\min }\right|\right)\right|\right)^{-1},
$$

Numerical examples demonstrate that the bound is more accurate and less dependent on the mesh non-uniformity than the previously available bounds.

\section{REFERENCES}

[1] Graham, I.G. and McLean, W. Anisotropic mesh refinement: the conditioning of Galerkin boundary element matrices and simple preconditioners. SIAM J. Numer. Anal., Vol. 44(4), pp. 1487-1513, (2006).

[2] Kamenski, L. Sharp bounds on the smallest eigenvalue of finite element equations with arbitrary meshes without regularity assumptions. Submitted for publication (in revision), arXiv:1908.03460v2, (2020). 